\title{
Erratum
}

\section{Rings in the haloes of planetary nebulae}

\author{
R. L. M. Corradi ${ }^{1}$, P. Sánchez-Blázquez ${ }^{2}$, G. Mellema ${ }^{3}$, C. Giammanco ${ }^{4}$, and H. E. Schwarz ${ }^{5}$ \\ ${ }^{1}$ Isaac Newton Group of Telescopes, Ap. de Correos 321, 38700 Sta. Cruz de la Palma, Spain \\ e-mail: rcorradi@ing.iac.es \\ 2 Departamento de Astrofísica, Universidad Complutense, 28040 Madrid, Spain \\ e-mail: pat@astrax.fis.ucm.es \\ 3 Sterrewacht Leiden, Postbus 9513, 2300 RA Leiden, The Netherlands \\ e-mail: mellema@strw.LeidenUniv.nl \\ ${ }^{4}$ Instituto de Astrofísica de Canarias, 38200 La Laguna, Tenerife, Spain \\ e-mail: corrado@ll.iac.es \\ 5 Cerro Tololo Inter-American Observatory, NOAO-AURA, Casilla 603, La Serena, Chile \\ e-mail: hschwarz@ctio.noao.edu
}

A\&A, 417, 637-646 (2004), 10.1051/0004-6361:20034420

Key words. ISM: planetary nebulae: general - stars: AGB and post-AGB - errata, addenda

Giammanco, C. should replace Gianmanco, C. in the authors' list. 